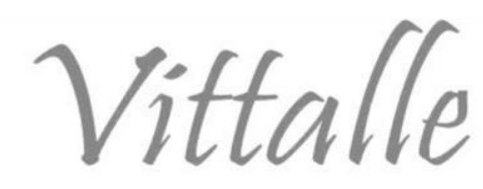

\title{
Efeitos da prematuridade sobre a aprendizagem
}

\author{
Mariana Gauterio Tavares ${ }^{\mathrm{a}^{*}}$, Jerusa Pires Pozzada ${ }^{\mathrm{a}}$, Augusto Duarte Faria ${ }^{\mathrm{b}}$, \\ Rui Alexandre Alves ${ }^{\mathrm{c}}$
}
${ }^{a}$ Hospital Universitário Dr. Miguel Riet Corrêa Jr. - HU/FURG/Ebserh, Rio Grande, RS, Brasil
bUniversidade Federal do Rio Grande - FURG, Rio Grande, RS, Brasil
${ }^{c}$ Faculdade de Psicologia e Ciências da Educação, Universidade do Porto, Porto, Portugal

\section{Histórico do Artigo \\ Recebido em: \\ 25/09/2019 \\ Aceito em: \\ $29 / 02 / 2020$ \\ Palavras-chave: \\ Prematuridade; \\ dificuldades de \\ aprendizagem; \\ cognição}

Keywords:

Prematurity; learning disabilities; cognition

\begin{abstract}
RESUMO
Considerando as relações encontradas na literatura sobre dificuldades de aprendizagem e nascimento prematuro, este estudo teve como objetivo avaliar as correlações entre indivíduos que nasceram muito prematuros e prematuros moderados/tardios com dificuldades de aprendizagem. Foi considerado o desempenho na realização de provas nas áreas de linguagem, aritmética, construção visuoespacial e leitura, além de variáveis como o peso, Apgar e tempo de internação em UTI Neonatal. Foram constituídos dois grupos de crianças entre 6 anos e 10 anos e 11 meses. Um grupo tinha histórico de nascimento entre 28 e 36 semanas de idade gestacional, e o outro de nascimento a termo (a partir de 37 semanas). Não foram encontradas diferenças significativas entre os grupos em relação ao desempenho nas tarefas. As correlações apontaram que o baixo peso ao nascer e o tempo de internação em centro de terapia intensiva não aparentam ser fator de risco para dificuldades de aprendizagem em crianças muito prematuras a prematuras moderadas/tardias. Estes resultados são vistos de forma positiva, pois permitem confiança nas habilidades das destas crianças e podem ajudar a reduzir o estigma da prematuridade.
\end{abstract}

Effects of prematurity on learning

\begin{abstract}
Considering the relationships found in the literature on learning disabilities and premature births, this study aimed to evaluate the correlations between individuals who were born very premature and moderate / late preterm with learning disabilities. It was considered the performance in tests in the areas of language, arithmetic, visuospatial construction and reading, as well as variables such as weight, Apgar and length of stay in neonatal ICU. Two groups of children between 6 years and 10 years and 11 months were constituted. One group had a history of birth between 28 and 36 weeks of gestational age, and the other with full term birth (from 37 weeks). No significant differences were found between groups regarding task performance. Correlations indicated that low birth weight and length of stay in intensive care unit do not appear to be a risk factor for learning disabilities in very preterm to moderate / late preterm infants. These results are viewed positively because they allow confidence in the abilities of premature children and can help reduce the stigma of prematurity.
\end{abstract}

\section{Introdução}

A Organização Mundial de Saúde (OMS) define como prematuras as crianças que nascem antes de completar 37 semanas de idade gestacional (IG), classificando-as como: prematuras extremas (aquelas com menos de 28 semanas), muito prematuras (entre 28 e 31 semanas e 6 dias) e prematuras moderadas a tardias (de 32 a 36 semanas e 6 dias) (1). A prematuridade é um tema que tem recebido cada vez mais atenção por associar-se com disfunções relacionadas à aprendizagem, problemas visuais, auditivos, motores, maior ìndice de déficit nas funções executivas, mais alto risco de doenças físicas e morte, além de se tornarem crianças com menor autonomia e mais dificuldades na tomada de decisões

\footnotetext{
*Autor correspondente: marianatav@gmail.com (Tavares M.G.)
} 
(1-4). Estas dificuldades na tomada de decisão estão associadas com a maior superproteção que recebem dos pais devidos aos problemas na gestação e pós-parto (5).

Há atualmente um maior conhecimento sobre os fatores de risco para prematuridade como, por exemplo, hipertensão arterial, diabetes, infecções urinárias e genitais, obesidade, desnutrição, distúrbios emocionais, tabagismo, consumo de drogas e transferência de mais de um embrião em fertilizações in vitro (6). No entanto, há razões para esperar mais nascimentos prematuros, pois se percebe, por exemplo, o aumento das taxas de gravidezes múltiplas, associadas a tratamentos para infertilidade e, posteriormente, idade materna avançada no parto (7).

A relação entre dificuldades de aprendizagem em prematuros extremos já é clara na literaura (8-10), porém, em relação aos demais graus de prematuridade as pesquisas apresentam muitas limitações. A principal encontra-se no fato de os grupos experimentais terem como critério único as crianças terem nascido com menos de 37 semanas de IG. Além disso, os estudos não referem os percentuais com que cada grau de prematuridade aparecem $(3,11,12)$. As pesquisas apresentam ainda baixo tamanho das amostras, utilização de instrumentos não padronizados e ausência de grupos controle (13).

Considerando as particularidades da prematuridade não extrema, a expectiva de mais nascimentos prematuros no futuro e buscando suprir algumas limitações da literatura, este estudo justifica-se por contribuir para formação de profissionais que lidam com esta população, tanto no contexto de follow-up de egressos de UTI (Unidade de Terapia Intensiva) Neonatal como no contexto educacional. Dessa forma, o objetivo deste trabalho foi avaliar as correlações entre indivíduos que nasceram muito prematuros e prematuros moderados/tardios com dificuldades de aprendizagem.

\section{Método}

A investigação aqui apresentada foi realizada com enfoque quantitativo, empregandose um plano de estudo comparativo-correlacional, a partir de dois grupos denominados G1 (crianças nascidas muito prematuras a prematuras moderadas e tardias) e G2 (crianças nascidas a termo). Foram considerados o desempenho na realização de provas nas áreas de linguagem, aritmética, construção visuoespacial e leitura, além de variáveis como peso, Apgar e tempo de internação em UTI. O local de estudo foi o Ambulatório Central do Hospital Universitário Dr. Miguel Riet Corrêa Jr. (HU-FURG/Ebserh).

\subsection{Participantes}

Foram utilizados dois métodos para seleção dos participantes, a fim de que a constituição final dos grupos fosse homogênea.

Para a composição dos participantes do G1 foi utilizado o método de amostragem aleatória, onde se considerou todos os 575 nascimentos prematuros no HU/FURG/Ebserh entre 01/11/2007 até 31/12/2012 e não ocorreram óbitos até o momento da alta, conforme os registros do hospital. Dessa forma, 181 famílias foram procuradas através de contato telefônico, sendo 75 números inexistentes/desatualizados, 30 que estavam ocupados ou não atenderam, 11 sujeitos excluídos e 3 que escolheram não participar. Além disto, 8 não compareceram.

Já o G2 foi composto por uma amostragem aleatória estratificada com uma lista dos pacientes que consultaram no Ambulatório de Pediatria do HU/FURG/Ebserh no ano anterior ao início da coleta (entre Junho de 2017 e Maio de 2018), considerando as variáveis idade e sexo. Neste grupo 29 sujeitos precisaram ser substituídos pelos 
seguintes motivos: 2 encontravam-se nos critérios de exclusão, 3 não aceitaram participar do estudo, 8 números estavam ocupados ou não atendiam, 4 famílias não poderiam participar na data da recolha de dados e 12 números eram inexistentes/desatualizados.

Os critérios de exclusão considerados foram: estudar em escola de educação especial, por já possuírem dificuldade de aprendizagem diagnosticada; problemas auditivos e; problemas visuais que comprometessem os resultados dos instrumentos. O critério de inclusão foi ter entre 06 anos e 10 anos e 11 meses.

\subsection{Instrumentos}

- Histórico clínico para coleta de informações do prontuário.

- Questionário clínico, sociodemográfico e educacional.

- Repetição de dígitos na ordem inversa - Retirada do NEUPSILIN - Inf (de Salles et al., 2016).

- Provas de Nomeação de figuras - Retiradas do NEUPSILIN - Inf (de Salles \& et al., 2016).

- Teste de Fluência Verbal Semântica categoria animais (Boston \& et al, 1996)

- Teste de Fluência Verbal Fonêmica F.A.S. (Delis, Kaplan \& Kramer, 2002)

- Subteste de Aritmética - Retirado da Weschler Intelligence Scale for Childen (WISC-IV)

- (Weschler, 1991, adaptado por Santos, Noronha, Rueda, Sisto e Casto, 2011).

- Construção Visuoespacial A - NEUPSILIN - Inf (de Salles et al., 2016)

- Construção Visuespacional B - Memória de Trabalho Operacional/ Prova dos blocos

- Retirada do NEUPSILIN - Inf (de Salles et al., 2016).

- Protocolo de Avaliação da Leitura de Palavras/Pseudopalavras Isoladas - LPI (de Salles, Piccolo, Zamo \& Toazza, 2013).

\subsection{Procedimentos}

Foi obtida a aprovação do Comitê de Ética e Pesquisa na Área da Saúde (CEPAS/FURG/Ebserh), em 05 de Junho de 2018, através do Parecer No 84/2018. O contato inicial com os participantes foi feito por telefonema, e consistiu numa explicação sumária do propósito do estudo. Os dados foram recolhidos em um único momento, e foram aplicados sempre na mesma ordem por uma profissional treinada, após assinatura do Termo de Assentimento Livre e Esclarecido e do Termo de Consentimento Livre e Esclarecido. Foi utilizada uma sala ampla e silenciosa e cada aplicação foi individual com duração média de 45 minutos. Posteriormente, os dados foram analisados no software estatístico IBM SPSS versão 25, onde foram realizadas as análises das frequências; Teste de Fischer; o teste paramétrico t de Student e coeficiente de correlação de Pearson.

\section{Resultados}

Participaram deste estudo 109 crianças entre 06 anos e 10 anos e 11 meses, e seus responsáveis.

O G1 foi composto por 54 crianças com idade média de 8,4 anos ( $\mathrm{DP}=1,5)$, nascidas prematuras, sendo $19,2 \%$ muito prematuras e $80,8 \%$ prematuras moderadas/tardias; $42,6 \%$ do sexo feminino e $57,4 \%$ do sexo masculino. Este grupo se caracterizou por apresentarem baixo peso ao nascer $(\mathrm{M}=1889 \mathrm{~g}, \mathrm{DP}=635,2)$ e Apgar significativamente menor ao primeiro e ao quinto minuto de vida quando comparado ao G2. Também apresentaram maior tempo 
de internação em UTI após o parto, além de mais problemas gestacionais tanto relacionados às progenitoras quanto às crianças e mais problemas pós-parto (Tabelas 1 e 2). Em média, as crianças do G1 estudavam no segundo ano das séries iniciais $(\mathrm{DP}=1,54)$, apesar de maior predominância no primeiro ano $(26,9 \%)$ (Tabela 3$)$.

O G2 foi composto por 55 crianças com média de idade de 8,3 anos ( $\mathrm{DP}=1,5)$, nascidas a termo com média de peso de 3299,1g ( $\mathrm{DP}=503,4)$ (Tabela 1), sendo 41,8\% do sexo feminino e $58,2 \%$ do sexo masculino (Tabela 2). Em média, o grupo estava no segundo ano das séries iniciais $(\mathrm{DP}=1,62)$, apesar de maior predominância no primeiro ano $(30,9 \%)$ (Tabela 3$)$.

Tabela 1 - Diferenças entre os grupos testados com Teste-t de Student

\begin{tabular}{lcccccccc}
\hline & \multicolumn{2}{c}{ G1 } & \multicolumn{9}{c}{ G2 } & t & p \\
\hline & Média & DP & N & Média & DP & N & & \\
\hline Idade (anos) & 8,4 & 1,5 & 54 & 8,3 & 1,5 & 55 & 0,29 & 0,77 \\
Escolaridade (anos) & 2,3 & 1,5 & 53 & 2,3 & 1,6 & 55 & 0,16 & 0,88 \\
$\begin{array}{l}\text { Idade gestacional } \\
\text { (semanas) }\end{array}$ & 33,2 & 2,6 & 54 & 39,6 & 1,3 & 49 & $-15,58$ & $<0,01^{*}$ \\
Peso (gramas) & 1889,0 & 635,2 & 54 & 3299,1 & 503,4 & 52 & $-12,64$ & $<0,01^{*}$ \\
Apgar 1" & 6,7 & 1,8 & 41 & 7,6 & 1,5 & 36 & $-2,39$ & $0,02^{*}$ \\
Apgar 5” & 8,2 & 1,1 & 42 & 9,0 & 0,8 & 42 & $-3,50$ & $<0,01^{*}$ \\
Tempo CTI & 27,9 & 25,4 & 53 & 0,1 & 0,4 & 55 & 8,32 & $<0,01^{*}$ \\
\hline Nota: p > 0,5 não significativo; 0,01 a 0,05 significativo. & & & & & &
\end{tabular}

Tabela 2 - Caracterização dos grupos

\begin{tabular}{|c|c|c|c|c|c|}
\hline & \multicolumn{2}{|c|}{ G1 } & \multicolumn{2}{|c|}{ G2 } & \multirow[t]{2}{*}{$\mathbf{p}$} \\
\hline & $\mathbf{N}$ & $\%$ & $\mathbf{N}$ & $\%$ & \\
\hline Sexo & & & & & 1,00 \\
\hline Feminino & 23 & 42,6 & 23 & 41,8 & \\
\hline Masculino & 31 & 57,4 & 32 & 58,2 & \\
\hline $\begin{array}{l}\text { Problemas } \\
\text { Gestacionais Maternos }\end{array}$ & & & & & $<0,01 *$ \\
\hline Sim & 46 & 85,2 & 23 & 41,8 & \\
\hline Não & 8 & 14,8 & 32 & 58,2 & \\
\hline $\begin{array}{l}\text { Problemas } \\
\text { Gestacionais do Bebé }\end{array}$ & & & & & $0,01 *$ \\
\hline Sim & 7 & 13,0 & 0 & 0,0 & \\
\hline Não & 47 & 87,0 & 55 & 100,0 & \\
\hline Problemas Pós-Parto & & & & & $<0,01 *$ \\
\hline Sim & 32 & 59,3 & 5 & 9,1 & \\
\hline Não & 22 & 40,7 & 50 & 90,9 & \\
\hline Internamento em CTI & & & & & $<0,01 *$ \\
\hline Sim & 50 & 90,6 & 2 & 3,6 & \\
\hline Não & 4 & 7,4 & 53 & 96,4 & \\
\hline
\end{tabular}


Tabela 3 - Escolaridade por frequências

\begin{tabular}{ccccc}
\hline & G1 & & G2 & \\
\hline & Frequência & Porcentagem & Frequência & Porcentagem \\
\hline Pré-escola & 6 & $11,3 \%$ & 7 & $12,7 \%$ \\
$1^{\circ}$ ano & 14 & $26,4 \%$ & 17 & $30,9 \%$ \\
$2^{\circ}$ ano & 9 & $17 \%$ & 6 & $10,9 \%$ \\
$3^{\circ}$ ano & 10 & $18,9 \%$ & 10 & $18,2 \%$ \\
$4^{\circ}$ ano & 9 & $17 \%$ & 10 & $18,2 \%$ \\
$5^{\circ}$ ano & 5 & $9,4 \%$ & 4 & $7,3 \%$ \\
$6^{\circ}$ ano & 0 & $0 \%$ & 1 & $1,8 \%$ \\
Total & 53 & $100 \%$ & 55 & $100 \%$ \\
Omissos & 1 & $0 \%$ & 0 & $0 \%$ \\
\hline
\end{tabular}

Os grupos apresentaram resultados semelhantes na realização dos testes quanto ao escore total (Tabela 4), com diferenças apenas no que se refere ao maior número de intrusões na Prova de Repetição de Dígitos na Ordem Inversa, onde o grupo de prematuros obteve maior número de erros. Nesta prova as intrusões se referem a quando a criança responde dígitos não presentes na sequência original ou evoca novamente um dígito mencionado na sequência original).

Estes resultados não foram entendidos como importantes neste estudo, pois a Prova de Repetição de Dígitos é um instrumento para avaliar a memória operatória, bem como a Prova de Construção Visuoespacial B e não foram encontradas alterações visuoespaciais ou nos demais subitens da Repetição de Dígitos. Tarefas afetadas pela memória operatória como linguagem ou habilidades aritméticas também não sofreram alterações quanto aos resultados das provas aplicadas.

Quanto as correlações, foi utilizada a interpretação de Cohen (14), onde valores entre 0,10 e 0,29 são considerados pequenos, entre 0,30 e 0,49 são considerados médios e acima de 0,5 são interpretados como grandes. Foi identificado que o baixo peso ao nascer e o tempo de internação em UTI não apareceram relacionados com 0 desempenho nos testes (Tabela 5).

Tabela 4 - Desempenho dos Grupos por instrumentos e suas categorias

\begin{tabular}{lcccccccc}
\hline & \multicolumn{9}{c}{ G1 } & \multicolumn{9}{c}{ G2 } & & t & p \\
\hline & Média & DP & N & Média & DP & N & & \\
\hline Nomeação de Figuras & & & & & & & & \\
Total de Acertos & 8,6 & 1,6 & 54 & 8,4 & 1,7 & 55 & 1,127 & 0,47 \\
Erros Fonológicos & 0,1 & 0,4 & 54 & 0,4 & 1,3 & 55 & $-1,624$ & 0,11 \\
Erros Semânticos & 0,1 & 0,3 & 54 & 0,2 & 0,4 & 55 & $-1,187$ & 0,24 \\
Repetição de Dígitos na & & & & & & & & \\
Ordem Inversa & & & & & & & & \\
Total de Acertos & 13,1 & 7,3 & 54 & 13,4 & 8,0 & 55 & $-0,17$ & 0,86 \\
Maior Sequência & 2,8 & 1,4 & 54 & 2,8 & 1,4 & 55 & $-0,08$ & 0,93 \\
Intrusões & 2,0 & 2,0 & 54 & 1,1 & 1,4 & 55 & 2,87 & $0.01 *$ \\
Inversões & 1,1 & 1,3 & 54 & 1,6 & 1,8 & 55 & $-1,53$ & 0,13 \\
Omissões & 3,5 & 3,6 & 54 & 2,8 & 2,8 & 55 & 1,14 & 0,26 \\
Trocas de Posição & 3,4 & 2,9 & 54 & 2,8 & 2,3 & 55 & 1,10 & 0,27 \\
\hline
\end{tabular}


M. G. Tavares et al./ Vittalle v. 32, n. 2 (2020) 8-16

Continuação

\begin{tabular}{|c|c|c|c|c|c|c|c|c|}
\hline & \multicolumn{2}{|c|}{ G1 } & \multicolumn{4}{|c|}{ G2 } & \multirow[t]{2}{*}{$\mathbf{t}$} & \multirow[t]{2}{*}{$\mathbf{p}$} \\
\hline & Média & DP & $\mathbf{N}$ & Média & DP & $\mathbf{N}$ & & \\
\hline \multicolumn{9}{|l|}{$\begin{array}{l}\text { Fluência Verbal } \\
\text { Semántica }\end{array}$} \\
\hline Total de Acertos & 9,7 & 3,7 & 54 & 9,2 & 3,5 & 54 & 0,609 & 0,54 \\
\hline \multicolumn{9}{|l|}{$\begin{array}{l}\text { Fluência Verbal } \\
\text { Fonológica }\end{array}$} \\
\hline FVF Total de Acertos & 10,5 & 7,3 & 54 & 10,2 & 7,7 & 54 & 0,244 & 0,81 \\
\hline FVF - Letra F & 3,9 & 3,2 & 54 & 3,4 & 3,1 & 54 & 0,768 & 0,44 \\
\hline FVF - Letra A & 3,9 & 2,5 & 54 & 3,4 & 2,4 & 54 & 1,067 & 0,29 \\
\hline FVF - Letra - S & 3,1 & 2,5 & 54 & 3,4 & 2,8 & 54 & $-0,436$ & 0,66 \\
\hline \multicolumn{9}{|l|}{ Aritmética } \\
\hline Total de Acertos & 12,1 & 3,4 & 52 & 11,2 & 4,0 & 55 & 1,237 & 0,22 \\
\hline \multicolumn{9}{|l|}{$\begin{array}{l}\text { Construção } \\
\text { Visuoespacial A }\end{array}$} \\
\hline Total de Acertos & 16,6 & 5,0 & 54 & 17,4 & 3,3 & 55 & $-0,973$ & 0,33 \\
\hline Tempo Total & 126,7 & 63,7 & 54 & 124,7 & 65,2 & 55 & 0,209 & 0,87 \\
\hline Quadrado & 4,9 & 1,5 & 54 & 5,1 & 1,0 & 55 & 0,815 & 0,42 \\
\hline Losango & 3,9 & 1,6 & 54 & 4,1 & 1,1 & 55 & 0,997 & 0,32 \\
\hline Margarida & 4,9 & 1,6 & 54 & 5,1 & 1,1 & 55 & $-0,793$ & 0,43 \\
\hline Figura Dupla & 3,0 & 1,4 & 54 & 3,1 & 1,3 & 55 & $-0,356$ & 0,72 \\
\hline \multicolumn{9}{|l|}{$\begin{array}{l}\text { Construção } \\
\text { Visuoespacial B }\end{array}$} \\
\hline Total de Acertos & 13,1 & 9,1 & 54 & 13,5 & 9,5 & 55 & $-0,213$ & 0,83 \\
\hline Maior Sequência & 3,3 & 1,3 & 54 & 3,4 & 1,5 & 55 & $-0,242$ & 0,81 \\
\hline \multicolumn{9}{|l|}{$\begin{array}{l}\text { Leitura de Palavras e } \\
\text { Pseudopalavras } \\
\text { Isoladas }\end{array}$} \\
\hline $\begin{array}{l}\text { (Total de Acertos } \\
\text { Palavras }\end{array}$ & 42,9 & 18,2 & 36 & 46,1 & 14,6 & 37 & $-0,821$ & 0,42 \\
\hline $\begin{array}{l}\text { Percentil por idade } \\
\text { (palavras) }(\mathrm{n}=73)\end{array}$ & 33,2 & 26,8 & 37 & 33,2 & 26,6 & 37 & $-0,008$ & 0,99 \\
\hline $\begin{array}{l}\text { Percentil por } \\
\text { escolaridade (palavras) }\end{array}$ & 31,9 & 28,6 & 36 & 28,0 & 26,9 & 38 & 0,613 & 0,54 \\
\hline $\begin{array}{l}\text { Total de Acertos } \\
\text { Pseudopalavras }\end{array}$ & 14,0 & 6,2 & 36 & 15,0 & 4,8 & 37 & $-0,778$ & 0,44 \\
\hline $\begin{array}{l}\text { Percentil por Acertos } \\
\text { Pseudopalavras }\end{array}$ & 70,0 & 30,7 & 36 & 76,1 & 23,9 & 37 & $-0,947$ & 0,35 \\
\hline $\begin{array}{l}\text { Percentil por } \\
\text { Escolaridade } \\
\text { (Pseudopalavras) }\end{array}$ & 39,3 & 25,7 & 36 & 39,8 & 28,4 & 37 & $-0,084$ & 0,93 \\
\hline $\begin{array}{l}\text { Percentil por idade } \\
\text { (Pseudopalavras) }\end{array}$ & 37,9 & 28,2 & 36 & 35,1 & 27,4 & 37 & 0,0437 & 0,66 \\
\hline
\end{tabular}


Tabela 5 - Correlações de Pearson entre as variáveis

\begin{tabular}{|c|c|c|c|c|c|c|c|c|c|c|c|c|}
\hline & Peso & $\begin{array}{c}\text { Tempo em } \\
\text { CTI }\end{array}$ & Nomeação & Repetição & FVS & FVF & Aritmética & $\begin{array}{l}\mathrm{CVb} / \\
\mathrm{OP}\end{array}$ & $\begin{array}{c}\text { CVa/ } \\
\text { Figuras }\end{array}$ & $\begin{array}{c}\text { CVa - } \\
\text { Tempo }\end{array}$ & $\begin{array}{c}\text { LPI - } \\
\text { Palavras }\end{array}$ & $\begin{array}{c}\text { LPI - } \\
\text { Pseudo } \\
\text { palavras }\end{array}$ \\
\hline Peso & 1.00 & & & & & & & & & & & \\
\hline $\begin{array}{c}\text { Tempo em } \\
\text { CTI }\end{array}$ & $-0.82^{* *}$ & 1.00 & & & & & & & & & & \\
\hline Nomeação & -0.16 & 0.13 & 1.00 & & & & & & & & & \\
\hline Repetição & 0.23 & -0.19 & -0.01 & 1.00 & & & & & & & & \\
\hline FVS & 0.03 & -0.14 & 0.03 & $0.46^{*}$ & 1.00 & & & & & & & \\
\hline FVF & 0.17 & -0.10 & 0.03 & $0.48^{*}$ & $0.32 *$ & 1.00 & & & & & & \\
\hline Aritmética & 0.16 & -0.12 & -0.08 & $0.38^{*}$ & 0.26 & $0.56^{* *}$ & 1.00 & & & & & \\
\hline $\mathrm{CVb} / \mathrm{OP}$ & 0.17 & -0.07 & 0.05 & $0.69 * *$ & $0.43^{*}$ & $0.33^{*}$ & $0.37 *$ & 1.00 & & & & \\
\hline $\mathrm{CVa}$ /Figuras & 0.16 & -0.17 & -0.04 & $0.45^{*}$ & $0.43^{*}$ & $0.33^{*}$ & $0.44 *$ & $0.47 *$ & 1.00 & & & \\
\hline $\begin{array}{c}\text { CVa/Figuras } \\
\text { - Tempo }\end{array}$ & 0.03 & -0.04 & $-0.54 * *$ & 0.15 & -0.14 & 0.07 & 0.11 & 0.18 & -0.01 & 1.00 & & \\
\hline $\begin{array}{c}\text { LPI - } \\
\text { Palavras }\end{array}$ & 0.18 & -0.13 & -0.02 & $0.50^{*}$ & $0.34 *$ & $0.61^{* *}$ & $0.61 * *$ & $0.48 *$ & $0.46^{*}$ & 0.14 & 1.00 & \\
\hline $\begin{array}{c}\text { LPI - } \\
\text { Pseudo } \\
\text { palavras }\end{array}$ & 0.13 & -0.09 & 0.02 & $0.46^{*}$ & $0.32 *$ & $0.56^{* *}$ & $0.52 *$ & $0.42 *$ & $0.44 *$ & 0.15 & $0.94 * *$ & 1.00 \\
\hline
\end{tabular}

\section{Discussão}

Este estudo teve como objetivo avaliar as correlações entre indivíduos que nasceram muito prematuros e prematuros moderados/tardios com dificuldades de aprendizagem. Foi verificado que tanto crianças prematuras não extremas quanto crianças nascidas a termo apresentaram desempenho semelhante na realização das provas aplicadas. Cabe salientar que o mesmo número de participantes foi excluído em ambos os grupos por já estudar em escola de educação especial, o que indica os grupos foram constituídos homogeneamente.

As análises estatísticas realizadas não mostraram diferenças significativas no desempenho dos grupos comparados, exceto em relação ao número de intrusões do teste de Repetição de Dígitos na Ordem Inversa. Este resultado é similar ao de Lundequist et al. (15) que, ao avaliarem um grupo de adolescentes muito prematuros, também não encontraram diferenças quando os compararam com adolescentes nascidos a termo (15).

A presente pesquisa foi realizada com crianças que apresentaram em média baixo peso ao nascer $(>1500 \mathrm{~g}$ e $<2500 \mathrm{~g}$ ) e não foram encontradas diferenças quando comparadas ao G2 de peso considerado normal (>2500). Diferencia-se portanto de estudos como o de Rodrigues, Mello e Fonseca (16) que encontraram pior desempenho em prematuros ao considerar somente crianças com extremo baixo peso $(<1500 \mathrm{~g})$. Este dado pode sugerir que apenas o baixo peso ao nascer pode não comprometer a aprendizagem da criança.

O baixo peso é uma característica do nascimento prematuro, bem como, em geral, quanto mais cedo o nascimento, maior o tempo de internação em UTI. No entanto, as correlações mostraram que o baixo peso ao nascer e o tempo de internação em UTI não influenciaram no desempenho dos testes. Este resultado se mostra bastante positivo principalmente para pais e professores de crianças muito prematuras a prematuras 
moderadas/tardias ao indicar que tais variáveis não aparentam ser fator de risco para dificuldades de aprendizagem em prematuros que nascem após 28 semanas, podendo auxiliar famílias a não perpetuarem o estigma da prematuridade, pois indica que estas crianças podem ter um desempenho normal como as nascidas a termo em relação à aprendizagem.

Caroni e Bastos (5) estudaram adolescentes que nasceram prematuros e relataram que as dificuldades que iniciaram na sala de parto, ou até mesmo durante o pré-natal, a perda das expectativas e idealizações sobre o nascimento do filho saudável e o período de internação em UTI faz com que os pais construam uma "redoma de vidro" em torno destas crianças, dificultando inclusive a autonomia e tomada de decisões no futuro. Porém, quando o nascimento prematuro ocorre a partir das 28 semanas de IG não costuma deixar sequelas físicas. Sendo assim, com o acompanhamento pediátrico indicando que a criança está saudável, os pais podem deixar de viver com "a sombra" do nascimento prematuro, focando mais nas capacidades dos seus filhos, e não mais tentando compensar dificuldades do desenvolvimento como se todas fossem relacionadas ao nascimento.

Outra questão importante a ser observada é que a maior parte das pesquisas acerca das dificuldades de aprendizagem apresentadas sobre prematuros são ainda realizadas com prematuros extremos. Dessa forma, apesar desta pesquisa utilizar uma outra população, este estudo limita-se também por ter agrupado os muitos prematuros junto aos prematuros tardios para rastrear seus déficts devido à dificuldade de encontrar estas crianças já em idade escolar. Sugere-se que, em futuros estudos, prematuros extremos, muito prematuros e prematuros tardios possam ser avaliados separadamente para que suas especificidades possam ser estudadas mais a fundo, apesar das dificuldades para constituição destes grupos.

Acredita-se que o fato de não haver prematuros extremos no G1 foi o que fez com o que os resultados dos grupos fossem semelhantes, diferindo dos resultados de outros estudos, como no caso de Rios-Flórez e Cardona-Agudelo (11), que estudaram crianças prematuras entre 6 e 10 anos, porém de um grupo mais heretogêneo, com os três graus de prematuridade. Os pesquisadores identificaram que crianças com antecedente de nascimento pré-termo aparesentavam dificuldades de aprendizagem em várias áreas de avaliação. Fan e Portuguez (4) também encontraram prematuridade como fator preditivo para risco em habilidades visuoespaciais, visuomotoras e verbais em um estudo transversal com crianças entre 6 e 7 anos. Porém as autoras também utilizaram prematuros extremos, muito prematuros e prematuros moderados/tardios no mesmo estudo. Ainda assim, classificaram estas crianças como tendo um nível intelectual considerado médio.

Para estudos futuros, sugere-se a inserção da prova de ditado, pois esta poderia avaliar problemas na consciência fonológica, considerada uma das principais dificuldades nos prematuros para Ríos-Flórez e Cardona-Agudelo (11). Além disso, um estudo multicêntrico com amostras maiores da população de prematuros nos diferentes graus, poderia revelar mais informações sobre os processos de aprendizagem $\mathrm{e}$ desenvolvimento dessas crianças.

Embora este tenha sido o primeiro estudo brasileiro a considerar apenas crianças de muito prematuras à prematuras tardias, deve-se ter cuidado ao generalizá-lo devido à especificidade da região onde foi realizado e ao pequeno tamanho da amostra. Mais estudos futuros sobre o desenvolvimento de prematuros devem clarificar nossa compreensão científica sobre esta população, melhorando a qualidade do atendimento em saúde e ensino oferecido.

\section{Conclusões}


Os resultados mostraram que crianças prematuras não extremas não apresentam dificuldades de aprendizagem quando comparadas a crianças nascidas a termo. Seus escores nos testes indicam que, em geral, podem ter o mesmo acompanhamento escolar durante os anos iniciais de escolarização, sugerindo que crianças que nasceram com IG a partir de 28 semanas não precisam carregar o estigma da prematuridade durante toda a infância no que se refere à aprendizagem. No entanto, cabe salientar a necessidade de mais pesquisas, principalmente estudos longitudinais e multicêntricos, com amostras maiores, que estudem os prematuros considerando especificidades de cada grau de prematuridade.

\section{Referências}

1. Organização Mundial da Saúde. Portal da Organização Mundial de Saúde. [Internet] 2017. Acesso em 15 de mar. de 2019. Disponível em: https://www.who.int/eportuguese/countries/bra/pt/

2. Von Doellinger P, Soares I, Sampaio A, Mesquita AR, Baptista J. Prematuridade, funções executivas e qualidade dos cuidados parentais: revisão sistemática de literatura. Psicologia: Teoria e Pesquisa 2017; 33:1-9.

3. Formiga CK, Martins R, Vieira, MEB, Linhares MBM. Avaliação do desenvolvimento de bebês nascidos pré-termo: a comparação entre idades cronológica e corrigida. Revista Brasileira de Crescimento e Desenvolvimento Humano 2015; 25(2): 230-6.

4. Fan RG, Portuguez MWP. Aprendizado e comportamento em crianças nascidas prematuras e com baixo peso em idade pré-escolar e em processo de alfabetização. Dissertação de Mestrado, Porto Alegre: 2008.

5. Caroni MM, Bastos OM. O adolescer prematuro: implicações da prematuridade no fortalecimento da autonomia [Dissertação de Mestrado]. Rio de Janeiro (RJ): Instituto Fernandes Figueira; 2012.

6. The American College of Obstetricians and Gynecologists. Practice bulletin no. 130: prediction and prevention of preterm birth. Obstetrics and Gynecology 2012; 120(4): 964-73

7. European Perinatal Health Report. Core indicators of the health and care of pregnant women and babies in Europe in 2015. [Internet]. Euro-Peristat Project, European Union ; [atualizado em 2018 Nov]. Disponível em: https://www.europeristat.com/images/EPHR2015_Euro-Peristat.pdf .

8. Akshoomoff N, Joseph RM, Taylor HG, Allred EN, Heeren T, O’Shea TM, Kuban KCK. Academic Achievement Deficits and Their Neuropsychological Correlates in Children Born Extremely Preterm. Journal of Developmental and Behavioral Pediatrics 2017; 38(8): 627-637.

9. Costa DS, Miranda DM, Burnett AC, Doyle LW, Cheong JLY, Anderson PJ. Executive Function and Academic Outcomes in Children Who Were Extremely Preterm. Journal of Pediatrics 2017; 140(3): 2017-2057

10. Leviton A, Joseph RM, Allred EN, O’Shea TM, Kuban KKC. Antenatal and neonatal antecedents of learning limitations in 10-year old children born extremely preterm. Early Human Development 2018; 118: 8-14.

11. Ríos-Flórez JA, Cardona-Agudelo V. Procesos de aprendizaje en niños de 6 a 10 años de edad con antecedente de nacimiento prematuro. Revista Latinoamericana de Ciencias Sociales, Niñez y Juventud 2016; 14(2): 1071-1085.

12. Rodrigues OMPR, Bolsoni-Silva, AT. Efeitos da prematuridade sobre o desenvolvimento de lactentes. Revista Brasileira Crescimento e Desenvolvimento Humano 2011; 21(1): 111-121.

13. Basso LA, Maia C P, Chula GVBPD, Arteche AX. Efeitos do nascimento pré-termo nas funções cognitivas de crianças: revisão sistemática. Revista Psicologia: Teoria e Prática 2016; 18(3): 98-114.

14. Cohen J. The significance of a product moment $r$. In Cohen J. Statistical power analysis for the behavioral sciences. Nova Iorque: Lawrence Erlbaum Associates; 1988. p. 75-108.

15. Lundequist A, Böhm B, Lagercrantz H, Forssberg H, Smedler A C. Cognitive outcome varies in adolescents born preterm, depending on gestational age, intrauterine growth and neonatal complications. Acta Paediatrica 2015; 104(3): 292-299.

16. Rodrigues MCC, Mello RR, Fonseca SC. Dificuldade de aprendizagem em escolares de muito baixo peso ao nascer. Jornal de Pediatria 2006; 82(1):6-14. 